\title{
APPLICATION OF HEDGE ALGEBRAS FOR CONTROLLING MECHANISMS OF RELATIVE MANIPULATION
}

\author{
Phan Bui Khoi ${ }^{1}$, Nguyen Van Toan ${ }^{2}$ \\ ${ }^{1}$ Hanoi University of Science and Technology, No.1 DaiCoViet Street, Hai Ba Trung, \\ Ha Noi, Viet Nam \\ ${ }^{2}$ Korea Institute of Science and Technology, 5 Hwarang-ro 14-gil, Seongbuk-gu, Seoul, \\ Republic of Korea
}

Email: khoi.phanbui@hust.edu.vn ${ }^{1}$,toan70411hd91@gmail.com ${ }^{2}$

Recieved: 7 November 2016; Accepted for publication: 18 July 2017

\begin{abstract}
This paper presents a method for controlling a mechanism of relative manipulation (MRM robot) that based on an algebraic approach to linguistic hedges in the fuzzy logic. The proposed model of MRM robot is introduced as a two-component mechanism of which two serial robots co-operate with each other to realize technological manipulations. MRM robot has complex structure; therefore, the robot system's mathematical equations describing dynamical behaviors are voluminous. Furthermore, the external components affect MRM robot's dynamics that are difficult to determine adequately and exactly. Applying the well-known methods (based on dynamical equations) such as PD/PID, computed torque algorithm etc. for robot control is difficult, especially with MRM robot. By dint of the human-like inference mechanism, designing controller via the fuzzy logic can overcome the mentioned drawbacks. However, the linguistic variables in the fuzzy logic are not represented by any physical values; and hence, the comparison among linguistic variables is unable. Moreover, the composition of fuzzy relations and the defuzzification use approximation functions which trigger error in data process. Hedge Algebras (HA) gives favorable conditions to restrict the fuzzy logic's drawbacks because the linguistic labels in Hedge Algebras are represented by the semantic values; and, the composition of fuzzy relations and the defuzzification are processed by simple interpolation and mapping functions. The obtained results from HA controller are compared to the obtained results from two methods which are fuzzy controller and computed torque controller.
\end{abstract}

Keywords: mechanism of relative manipulation (MRM robot), hedge algebras.

\section{INTRODUCTION}

The mechanism of relative manipulation (MRM) robot includes two serial/parallel robots which has complex structure [1,2]; thus, the robot's dymical equations are complicated $[3,4]$ and [5]. It causes the difficulty when applying classical algorithms for robot control. In [6], the fuzzy logic is applied to control MRM robot that overcomes the mentioned problem. Besides, 
the obtained results from fuzzy controller are compared with the obtained results from computed torque controller to evaluate the reliability. The comparison shows the benefits when applying the fuzzy logic to control MRM robot. However, the application of fuzzy logic still exists several limitations, namely the fuzzy logic's linguistic variables cannot be compared with each other, the use of fuzziness measure and composition of fuzzy relations is still complicated; the defuzzification must be processed by approximation functions; and hence, it leads to the error in implementation process. With specific features, Hedge Algebras (HA) can overcome aboved drawbacks. Firstly, the linguistic labels in HA are represented by the physical values (namely the semantic values of each linguistic label is calculated by the semantically quantifying mapping), so they can be compared with each other. Moreover, the input and output do not need the defuzzification because the semantically quantifying mapping of hedge algebras maps input/output's physical values to the value domain $[0,1]$ to process; and therefore, to calculate the physical values of controller's outputs, we just need to map their semantic value (belongs to $[0,1])$ to their physical domain. The calculation of output's semantic value is conducted by an interpolation function. The product operator and the average operator are usually used to transform m-demension SAM table (the table of the semantic relationship between inputs and outputs) to 2-dimension SAM table, and then building the interpolation function. By doing this, the composition of fuzzy relations, the fuzziness measure and the defuzzification are avoided. As a results, the complexity is reduced and the accuracy is enhanced. Several papers applied HA in control and obtained reasonable results, as [7 - 16]. However, applying HA to robot control is relatively new. This paper proposes a method for controlling a mechanism of relative manipulation that based on an algebraic approach to linguistic hedges in fuzzy logic. To evaluate the proposed method, the obtained results from HA controller are compared to the obtained results from two methods which are presented in [6].

\section{MRM ROBOT'S KINEMATIC AND DYNAMICAL MODEL}

Figure 1 presents 5-DOF MRM robot model which is used for welding (or lazer machining), including 3-DOF tool-robot (T), 2-DOF jig-robot (B), and technological object (W), (as presented in [6]). The tool-robot consists of unmovable base $\mathrm{L}_{10}$, movable links are denoted by $\mathrm{L}_{11}, \mathrm{~L}_{12}$ and $\mathrm{L}_{13}$. The jig-robot consists of unmovable base $\mathrm{L}_{20}$ and movable links $\mathrm{L}_{21}, \mathrm{~L}_{22}$.

\subsection{MRM robot's manipulation motion}

The motion of the links:

Links $\mathrm{L}_{11}$ and $\mathrm{L}_{12}$ rotate around axes $\mathrm{z}_{10}$ and $\mathrm{z}_{11}$ respectively which are performed by corresponding angles $\Theta_{11}$ and $\Theta_{12}$; link $L_{13}$ translates along axis $z_{12}$ which is performed by $d_{13}$.

Links $\mathrm{L}_{21}$ and $\mathrm{L}_{22}$ rotate around axes $\mathrm{z}_{20}$ and $\mathrm{z}_{21}$ respectively which are performed by corresponding angles $\Theta_{21}$ and $\Theta_{22}$.

The adequately generalized coordinates of MRM robot is presented by:

$$
\mathbf{q}=\left[\mathrm{q}_{1}, . ., \mathrm{q}_{5}\right]^{\mathrm{T}}=\left[\theta_{11}, \theta_{12}, \mathrm{~d}_{13}, \theta_{21}, \theta_{22}\right]^{\mathrm{T}}
$$

MRM robot's kinematic parameters are taken as given in [6] and presented in Table 1.

Parameters $d_{11}, a_{11}, a_{12}$ are kinematic dimensions of the tool-robot, $\Theta_{20}, d_{20}, a_{20}, \alpha_{20}$, are Denavit-Hartenberg parameters describing the position and the orientation of the jig-robot's base frame in the general base frame. 
Table 1. Parameters describe the robot structure.

\begin{tabular}{|c|c|c|c|c|c|c|c|}
\hline $\mathrm{d}_{11}$ & $\mathrm{a}_{11}$ & $\mathrm{a}_{12}$ & $\Theta_{20}$ & $\mathrm{~d}_{20}$ & $\mathrm{a}_{20}$ & $\alpha_{20}$ & $\mathrm{~d}_{22}$ \\
\hline $0.82(\mathrm{~m})$ & $0.33(\mathrm{~m})$ & $0.33(\mathrm{~m})$ & 0 & $0.3(\mathrm{~m})$ & $0.45(\mathrm{~m})$ & $-90^{0}$ & $0.1(\mathrm{~m})$ \\
\hline
\end{tabular}

During the implemention process of a technological manipulation, link $\mathrm{L}_{13}$ brings the tool performing the relative motion to the workpiece (set-up on movable jig-table $\mathrm{L}_{22}$ ). The welding is conducted by the tool's manipulation motions which work on the workpiece. These motions are represented through robot's manipulation coordinates in the moving space. The manipulation coordinates are determined by the position and the orientation of Catersian coordinates $\mathrm{x}_{13} \mathrm{y}_{13} \mathrm{Z}_{13}$ (on link $\mathrm{L}_{13}$ ) in Cartesian coordinates $\mathrm{x}_{22} \mathrm{y}_{22} \mathrm{Z}_{22}$ (on movable jig-table $\mathrm{L}_{22}$ ). The robot's manipuation coordinates are presented by:

$$
\mathbf{p}=\left[\mathrm{p}_{1}, \mathrm{p}_{2}, \ldots, \mathrm{p}_{6}\right]^{\mathrm{T}}=\left[{ }^{22} \mathrm{x}_{\mathrm{p} 13},{ }^{22} \mathrm{y}_{\mathrm{p} 13},{ }^{22} \mathrm{z}_{\mathrm{p} 13},{ }^{22} \alpha_{\mathrm{p} 13},{ }^{22} \beta_{\mathrm{p} 13},{ }^{22} \eta_{\mathrm{p} 13}\right]^{\mathrm{T}}
$$

The bottom right indexes of elements are represented by index of $\mathrm{L}_{13}$, the upper left indexes are represented by index of $\mathrm{L}_{22}$.

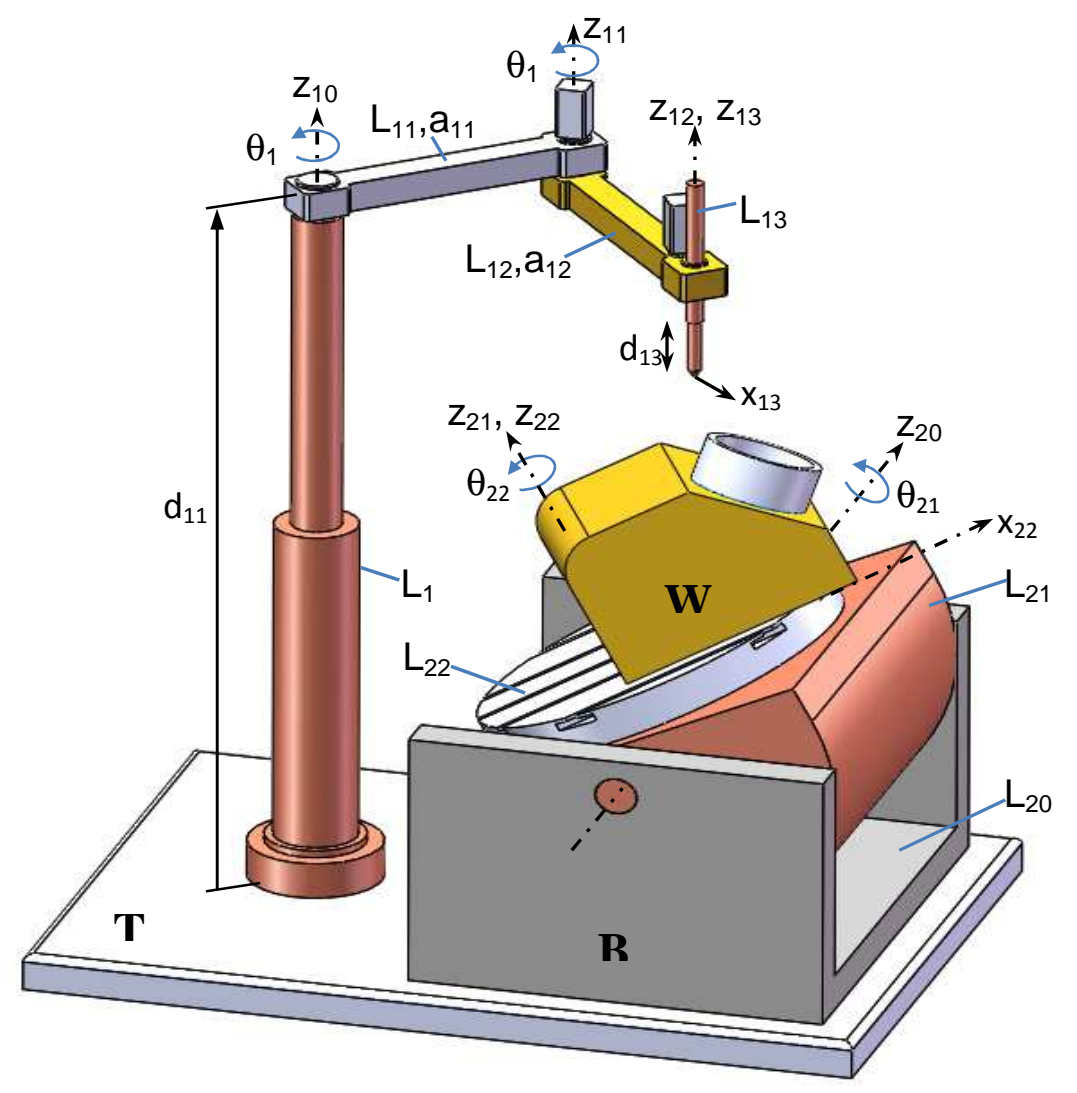

Figure 1. MRM robot - workpiece. 
Depending on the technological object and the kind of technological manipulation, the elements of the vector $\mathbf{p}$ are determined as the function of time or numeric data. As mentioned, to compare the proposed method with the computed torque controller and the fuzzy controller (as presented in [6]), we consider the technological manipulation that MRM robot welded tube part to machine part.

1. Jig-robot's table

2. Machine part

3. Tube

4. Welding path

5. Machine part's surface

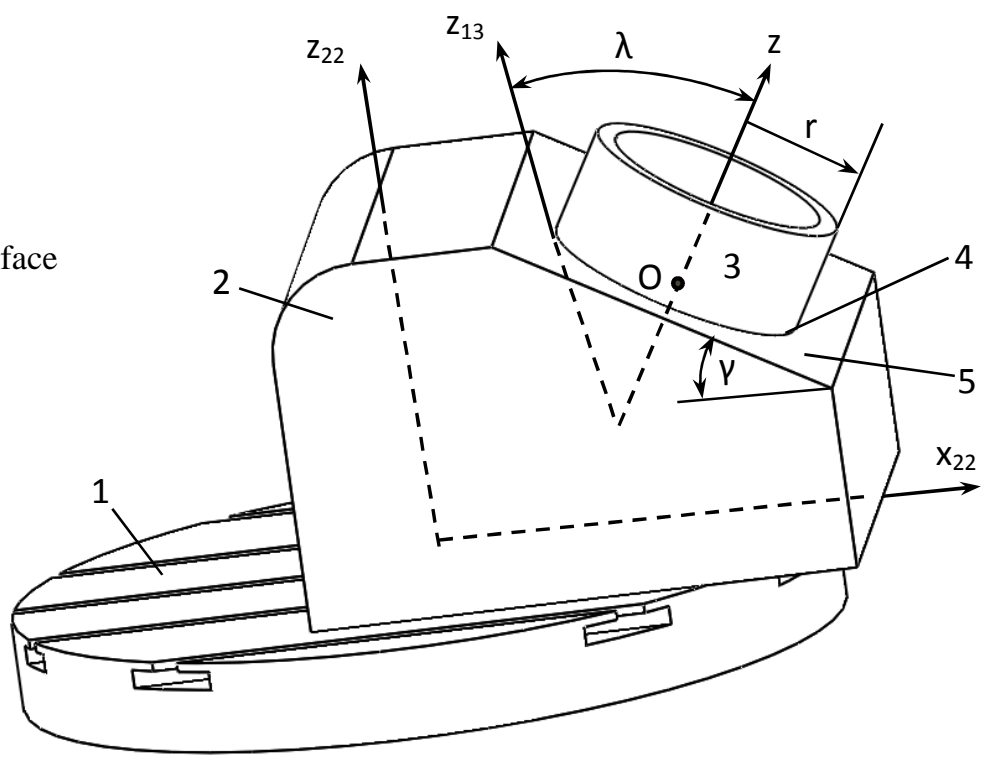

Figure 2. Welding path is realized by MRM robot.

The method of kinematic investigation in [6] is used for this paper; and, the welding process is shown in Figure 2. Machine part 2 is on jig-robot's Table 1 which is welded with tube 3 following butt-weld 4 , with parameters:

- The path of the butt-weld 4 is intersection of tube surface with machine part's surface 5, which is a parallel plane with $\mathrm{y}_{22}$ axis and slope an angle $\gamma$ to $\mathrm{x}_{22}$ axis.

- The tube axis $\mathrm{z}$ is perpendicular to the surface 5.

- Center $\mathrm{O}$ of welding path's circle have coordinates $\left(x_{0}, y_{0}, z_{0}\right)$ in the frame $x_{22} y_{22} z_{22}$, radius of the tube is $r$.

- The axis of welding tool (welding gun) $z_{13}$ is coplanar and sloping an angle $\lambda$ with tube axis.

- The velocity $\mathrm{v}_{\mathrm{h}}$ of welding tool's head along the welding path which is given based on the welding technics.

Table 2 shows the parameters appearing in this application which are the same in [6] exept for $\mathrm{v}_{\mathrm{h}}$ (in [6], $\mathrm{v}_{\mathrm{h}}=0.2 \mathrm{~m} / \mathrm{s}$ ).

Table 2. Parameters describe the machining object.

\begin{tabular}{|c|c|c|c|c|c|c|}
\hline$\gamma$ & $\mathrm{x}_{\mathrm{o}}$ & $\mathrm{y}_{\mathrm{o}}$ & $\mathrm{z}_{\mathrm{o}}$ & $\mathrm{r}$ & $\lambda$ & $\mathrm{v}_{\mathrm{h}}$ \\
\hline $30^{0}$ & $0.1(\mathrm{~m})$ & $0.1(\mathrm{~m})$ & $0.1(\mathrm{~m})$ & $0.07(\mathrm{~m})$ & $45^{0}$ & $0.0275(\mathrm{~m} / \mathrm{s})$ \\
\hline
\end{tabular}


By virtue of technological object, the technical requirements for welding manipulation as aboved and parameters in Table 2; the relative position of welding gun need to be determined, namely the elements of $\mathbf{p}$ (2.2). The kinematics and plan motion for robot is investigated by applying the same method in [2] and [6]. The obtained trajectory will be the input data (desired trajectory) for the controller. These data are saved to files Position.txt, Velocity.txt, Acceleration.txt which contain robot's trajectory, including corresponding position, velocity and acceleration.

\subsection{MRM robot's dynamical equations}

As given in [6], the robot's dynamical equations are writen in the matrix form:

$$
\mathbf{M}(\mathbf{q}) \ddot{\mathbf{q}}+\mathbf{C}(\mathbf{q}, \dot{\mathbf{q}})+\mathbf{G}(\mathbf{q})+\mathbf{Q}=\mathbf{U}
$$

where

$$
\begin{gathered}
\mathbf{M}(\mathbf{q})=\left[\sum_{\mathrm{i}=1}^{5}\left(\mathrm{~J}_{\mathrm{Ti}}^{\mathrm{T}} \mathrm{m}_{\mathrm{i}} \mathrm{J}_{\mathrm{Ti}}+\mathrm{J}_{\mathrm{Ri}}^{\mathrm{T}} \Theta_{\mathrm{ci}} \mathrm{J}_{\mathrm{Ri}}\right)\right]_{5 \times 5} \\
\mathbf{C}(\mathbf{q}, \dot{\mathbf{q}})=\left[\mathrm{c}_{1}, \mathrm{c}_{2}, \ldots, \mathrm{c}_{5}\right]^{\mathrm{T}}, \quad \mathrm{c}_{\mathrm{j}}=\sum_{\mathrm{k}, \mathrm{l}=1}^{5}(\mathrm{k}, \mathrm{l} ; \mathrm{j}) \dot{\mathrm{q}}_{\mathrm{k}} \dot{\mathrm{q}}_{\mathrm{l}}, \quad(\mathrm{k}, \mathrm{l} ; \mathrm{j})=\frac{1}{2}\left(\frac{\partial \mathrm{m}_{\mathrm{kj}}}{\partial \mathrm{q}_{\mathrm{l}}}+\frac{\partial \mathrm{m}_{\mathrm{lj}}}{\partial \mathrm{q}_{\mathrm{k}}}-\frac{\partial \mathrm{m}_{\mathrm{kl}}}{\partial \mathrm{q}_{\mathrm{j}}}\right) \\
\mathbf{G}(\mathbf{q})=\left[\mathrm{g}_{1}, \mathrm{~g}_{2}, . ., \mathrm{g}_{5}\right]^{\mathrm{T}}, \quad \mathrm{g}_{\mathrm{j}}=\frac{\partial \Pi}{\partial \mathrm{q}_{\mathrm{j}}}
\end{gathered}
$$

The values $\mathrm{m}_{\mathrm{i}}, \mathrm{J}_{\mathrm{Ti}}$ and $\mathrm{J}_{\mathrm{Ri}}$ are the mass, the translational Jacobian, and the rotational Jacobian respectively of link $\mathrm{I} ; \Theta_{\mathrm{ci}}$ is the inertia tensor of the $\mathrm{i}^{\text {th }}$ link about center of mass $\mathrm{C}_{\mathrm{i}}$ which is expressed in the frame with origin at the $i^{\text {th }}$ link's center of mass; $i=1, \ldots, 5 ; \Pi$ is the potential energy of the system; $\mathbf{q}, \dot{\mathbf{q}}, \ddot{\mathbf{q}}$ are vectors of the joint positions (2.1), velocities, accelerations, respectively; $(\mathrm{k}, \mathrm{l} ; \mathrm{j})$ is Christoffel notations; $\mathrm{m}_{\mathrm{kl}}(\mathrm{k}, \mathrm{l}=1, \ldots, 5)$ are elements of the mass matrix $\mathbf{M}(\mathrm{q})$.

The vector $\mathbf{U}$ is the expression of the calculated force/torque which matchs the programed motion of the robot $[17,18]$.

$$
\mathbf{U}=\left[\mathrm{U}_{1}, \mathrm{U}_{2}, . ., \mathrm{U}_{5}\right]^{\mathrm{T}}
$$

The vector $\mathbf{Q}$ is the expression of the generalized force of friction, the disturbance as well as other non-conservative forces applying on the robot.

$$
\mathbf{Q}=\left[\mathrm{Q}_{1}, \mathrm{Q}_{2}, . ., \mathrm{Q}_{5}\right]^{\mathrm{T}}
$$

In general, it is hard to build up exactly the robot's dynamical equations because of the difficulty of their determiniation. To solve these drawbacks, a method based on the fuzzy logic which is proposed in [6] for controlling MRM robot. However, the fuzzy method still exists some shortcomings. This paper proposes a method based on HA to overcome above drawbacks and restrict the shortcoming of the fuzzy controller.

We assume that $\mathbf{Q}$ can be determined exactly to have comparable base between the methods. The vector $\mathbf{Q}$ is the combination of friction and disturbance forces. It depends on the generalized position and the generalized velocity: 


$$
\begin{gathered}
\mathbf{Q}_{\mathrm{fr}}=\mu\left[\dot{\mathrm{q}}_{1}, \ldots, \dot{\mathrm{q}}_{5}\right]^{\mathrm{T}} \\
\mathbf{Q}_{\text {dis }}=\delta\left[\sin \left(\mathrm{q}_{1}\right)+1, \cos \left(\mathrm{q}_{2}\right)+1, \sin \left(\mathrm{q}_{3}\right), \sin \left(\mathrm{q}_{4}\right) \cos \left(\mathrm{q}_{4}\right), \sin \left(2 \mathrm{q}_{5}\right)\right]^{\mathrm{T}}
\end{gathered}
$$

then

$$
\mathbf{Q}=\mathbf{Q}_{\mathrm{fr}}+\mathbf{Q}_{\mathrm{dis}}
$$

with $\mu=0.003$ and $\delta=0.5$.

Based on this condition, the computed torque method is conducted to obtain the clear control results.

$\mathbf{Q}$ is not used for the fuzzy method and the HA method. The obtained results from three methods will be compared with each other to evaluate the HA controller.

\section{HEDGE ALGEBRAS CONTROLLER}

The technical requirements are described in the previous section which are the position, orientation and the velocity of the welding gun in welding technics. To control the welding gun following the desired welding path in jig-table's Cartesian coordinates, MRM robot's joints are controlled following their trajectory which are received from the inverse kinematics. Throughout this paper, $\mathrm{q}_{\mathrm{i}}(\mathrm{t})$ and $\dot{\mathrm{q}}_{\mathrm{i}}(\mathrm{t})$ present actuated joint's position and velocity of MRM robot, $q_{i}^{d}(\mathrm{t})$, $\dot{q}_{i}^{d}(\mathrm{t})$ present desired position and velocity.

The controller is designed so that $q_{i}(t)$ and $\dot{q}_{i}(\mathrm{t})$ follow $q_{i}^{d}(\mathrm{t})$ and $\dot{q}_{i}^{d}(\mathrm{t})$. By doing this, the welding gun follows the desired welding path in the Cartesian coordinate system of the jigrobot's table. In other words, the control purpose is to adjust the driving torque at actuated joints so that the position error $\boldsymbol{e}(t)$ and the velocity error $\dot{\boldsymbol{e}}(t)$ are small as desire.

The position error and the velocity error are computed by:

$$
\left\{\begin{array}{l}
\mathrm{e}_{\mathrm{i}}(\mathrm{t})=\mathrm{q}_{\mathrm{i}}(\mathrm{t})-\mathrm{q}_{\mathrm{i}}^{\mathrm{d}}(\mathrm{t}) \\
\dot{\mathrm{e}}_{\mathrm{i}}(\mathrm{t})=\dot{\mathrm{q}}_{\mathrm{i}}(\mathrm{t})-\dot{\mathrm{q}}_{\mathrm{i}}^{\mathrm{d}}(\mathrm{t})
\end{array}\right.
$$

In [6], the fuzzy controller is proposed for controlling MRM robot which aims to reduce the difficulty of the dynamical identification. In addition, the computed torque controller is used to compare with the fuzzy controller. This paper uses these methods to get the results which are used to compared with the obtained results from proposed controller. The control rules and the parameters of fuzzy controller and computed torque controller are similarly chosen in [6].

\subsection{Background of hedge algebras}

An algebra structure called hedge algebras - HA which is defined $A X=(\mathbf{X}, \boldsymbol{G}, \boldsymbol{H}, \leq)$ therein $\mathbf{X}=\operatorname{Dom}(X)$ is a term-set of a linguistic variable $X, \boldsymbol{G}=\left\{\mathbf{0}, \boldsymbol{c}, \boldsymbol{W}, \boldsymbol{c}^{+}, \boldsymbol{1}\right\}$ is the set of the primary terms of linguistic variables ( $\boldsymbol{W}$ is neutral element), $\boldsymbol{H}=\left\{\boldsymbol{h}^{-}, \boldsymbol{h}^{+}\right\}$is a set of unary operations representing linguistic hedges and " $\leq$ " is a partially ordering relation in $\mathbf{X}$.[12], [13].

If $\mathbf{X}$ and $\mathbf{H}$ are linearly ordered sets then $\mathrm{AX}$ is the linear hedge algebras. By dint of the action-effect of the hedges $h \in \mathbf{H}$ on element $x \in \mathbf{X}$, we observe $\mathbf{H}(\mathrm{x})$ is a set of all elements $\mathrm{hx} \in \mathbf{X}$ and $h x=h_{n} \ldots h_{1} x$ with $h_{n}, \ldots, h_{1} \in \mathbf{H}$; and therefore, $\mathbf{H}(\mathrm{x})$ is a linearly ordered set [14]. 
If $\mathbf{X}$ is created from $\mathbf{G}$ and hedges therein $\mathbf{G}$ is a linearly ordered set, then $\mathbf{X}$ is also a linearly ordered set. Moreover, with $\mathrm{u}$ and $\mathrm{v}$ belong to $\mathbf{X}$, if $u<v, u \notin \mathbf{H}(\mathrm{v})$ and $\mathrm{v} \notin \mathbf{H}(\mathrm{u})$, then $\mathbf{H}(\mathrm{u})<\mathbf{H}(\mathrm{v})$. In addition, if $u=h_{n} \ldots h_{1} x$ and $v=h_{m} \ldots h_{1} x(\mathrm{~m}<\mathrm{n})$, then $\mathrm{u}$ is more special than $\mathrm{v}$. Elements in $\mathbf{H}$ are comparable; and, if $h \neq k$ and $h x=k x$ then $\mathrm{x}$ is a fixed point [13], [14], [15].

Fuzziness measure: a given fuzziness measure of element $\tau$ called $f m(\tau), \tau \in \mathbf{X}$ that the values of $f m(\tau)$ always belong to $[0,1][16]$.

$$
\text { (1) } f m(\tau)=0 \text {, if } \tau \text { is clear. }
$$

(2) If $h$ is a hedge and $\tau$ is a fuzzy value then $h \tau$ is more featured than $\tau$, so we have

$$
f m(h \tau)<f m(\tau) \text { and } f m(h \tau)=\mu(h) f m(\tau) \text {, with } \forall \tau \in \mathbf{X} .
$$

(3) If $c+, c-$ are two primary terms in $\mathbf{X}$, then

$$
f m(c+)+f m(c-)=1 .
$$

Semantically quantifying mapping: $\mathrm{By} f \mathrm{~m}$ is a fuzziness measure of $\mathbf{X}$, semantically quantifying mapping $v: \mathbf{X} \rightarrow[0,1]$ is determined [12]:

$$
\begin{aligned}
& \text { (1) } \square(\mathbf{W})=\theta=\mathrm{fm}\left(\mathrm{c}^{-}\right), \square\left(\mathrm{c}^{-}\right)=\theta-\alpha \mathrm{fm}\left(\mathrm{c}^{-}\right)=\beta \mathrm{fm}\left(\mathrm{c}^{-}\right), \square\left(\mathrm{c}^{+}\right)=\theta+\alpha \mathrm{fm}\left(\mathrm{c}^{+}\right) \\
& \text {(2) } \square\left(\mathrm{h}_{\mathrm{j}} \mathrm{x}\right)=\square(\mathrm{x})+\operatorname{Sign}\left(\mathrm{h}_{\mathrm{j}} \mathrm{x}\right) \cdot\left\{\sum_{\mathrm{i}=\operatorname{Sign}(\mathrm{j})}^{\mathrm{fm}}\left(\mathrm{h}_{\mathrm{i}} \mathrm{x}\right)-\omega\left(\mathrm{h}_{\mathrm{j}} \mathrm{x}\right) \mathrm{fm}\left(\mathrm{h}_{\mathrm{j}} \mathrm{x}\right)\right\}
\end{aligned}
$$

where $\omega\left(h_{\mathrm{j}} \mathrm{x}\right)=\frac{1}{2}\left[1+\operatorname{Sign}\left(\mathrm{h}_{\mathrm{j}} \mathrm{x}\right) \operatorname{Sign}\left(\mathrm{h}_{\mathrm{p}} \mathrm{h}_{\mathrm{j}} \mathrm{x}\right)(\beta-\alpha)\right]$, and

$$
\mathrm{j} \in\{-\mathrm{q} \leq \mathrm{j} \leq \mathrm{p} \& \mathrm{j} \neq 0\}=[-\mathrm{q} \ldots \mathrm{p}] . \sum_{\mathrm{i}=-\mathrm{q}}^{-1} \mu\left(\mathrm{h}_{\mathrm{i}}\right)=\alpha \text { and } \sum_{\mathrm{i}=1}^{\mathrm{p}} \mu\left(\mathrm{h}_{\mathrm{i}}\right)=\beta,
$$

with $\alpha, \beta>0$ and $\alpha+\beta=1$.

$\mathbf{W}$ is neutral element.

In the fuzzy logic, the composition of fuzzy relations is demonstrated by $\mathrm{m}$ dimensional FAM table (the table of the fuzzy rules among the inputs and outputs), we will use the semantically quantifying mapping to convert FAM table to semantic table SAM.

\subsection{Applying hedge algebras for controlling MRM robot}

Step 1: Determining inputs/outputs

The inputs of hedge algebras controller including $\mathbf{q}^{\mathrm{d}}(\mathrm{t}), \dot{\mathbf{q}}^{\mathrm{d}}(\mathrm{t}), \mathbf{q}(\mathrm{t}), \dot{\mathbf{q}}(\mathrm{t})$ which are desired trajectory, velocity and real trajectory, velocity of MRM robot. The output is adjusted torque $\mathbf{u}(\mathrm{t})$ at actuated joints so that $\mathbf{q}(\mathrm{t}), \dot{\mathbf{q}}(\mathrm{t})$ follow $\mathbf{q}^{\mathrm{d}}(\mathrm{t}), \dot{\mathbf{q}}^{\mathrm{d}}(\mathrm{t})$, in other words, $\mathbf{e}$ and $\dot{\mathbf{e}}$ are small as desire:

$$
u_{i}=\tau_{i}-\tau_{i}^{d}
$$

here in $\tau_{i}$ is real driving torque set at the $\mathrm{i}^{\text {th }}$ actuated joints to $\mathrm{e}_{\mathrm{i}}$ and $\dot{e}_{l}$ come to $0 ; \tau_{i}^{d}$ is theoretical driving torque set at the $\mathrm{i}^{\text {th }}$ actuated joints to $\mathrm{e}_{\mathrm{i}}$ and $\dot{e}_{l}$ come to 0 .

Now, we can consider controller's inputs of $\mathbf{e}(\mathrm{t})$ and $\dot{\mathbf{e}}(\mathrm{t})$, output of $\mathbf{u}(\mathrm{t})$

$$
\begin{aligned}
& \mathbf{e}=\left[e_{1}, \ldots, e_{5}\right]^{T} \\
& \mathbf{d e}=\left[\dot{e}_{1}, \ldots, \dot{e}_{5}\right]^{T} \\
& \mathbf{u}=\left[u_{1}, \ldots, \mathrm{u}_{5}\right]^{T}
\end{aligned}
$$


where $e_{i}, \dot{e}_{i}, u_{i}$ are position error, velocity error and ajusted torque at the $\mathrm{i}^{\text {th }}$ actuated joint of MRM robot, respectively. Approximately physical domain of inputs/outputs are later used in Table 6.

Step 2: Choosing hedge parameters

Owning to the number of linguistic terms in the fuzzy controller, we chose hedge parameters so that each input/output $e_{i}, \dot{e}_{i}, u_{i}$ will be demostrated by five linguistic labels with five semantic values. This gives the same condition when designing two controllers for the purpose of comparing the proposed controller and the fuzzy controller. The set of the primary terms of linguistic variables and hedges are chosen such that if applying hedges to the set of the primary terms of linguistic variables, then we observe five linguistic values which present for each input/ output $e_{i}, \dot{e}_{i}, u_{i}$ of each joint:

$$
\begin{gathered}
\mathbf{G}=\{0, \mathrm{~S}, \mathrm{~W}, \mathrm{~B}, 1\} \text { with } \mathrm{S}=\text { Small; } \mathrm{B}=\text { Big. } \\
\mathbf{H}=\left\{\mathrm{H}^{-}, \mathrm{H}^{+}\right\} \text {with } \mathrm{H}^{-}=\mathrm{L} ; \mathrm{H}^{+}=\mathrm{V}=>\mathrm{q}=1, \mathrm{p}=1 \text { herein } \mathrm{L}=\text { Little; } \mathrm{V}=\text { Very. }
\end{gathered}
$$

Now, each input/output $e_{i}, \dot{e}_{i}, u_{i}$ are presented by one hedge algebras which is created from the set of the primary terms of the linguistic variable $\mathbf{G}$ and the set of hedges $\mathbf{H}$ through a linearly ordering relation. However, we just use the partition $\mathrm{k}=2$ when creating linguistic values in each hedge algebras which aims to compare with the fuzzy controller. So, the linguistic terms in hedge algebras are presented by $\mathbf{X}=\{$ VS, LS, W, LB, VB $\}$. In the fuzzy controller, the linguistic values are the same for inputs/ outputs, but their physical domains are different. In the same way, with each hedge algebras present $e_{i}, \dot{e}_{i}$ and $u_{i}$, their linguistic values are the same; and therefore, they will have the same semantic values. However, those values just describe semantics in individual input/ output's physical domains; and hence, by mapping their semantic values to their real physical domain, we observe different values for different inputs/ outputs.

The values 0 and 1 in $\mathbf{G}$ present elements which have the smallest semantic value and the biggest semantic value, respectively; and, they are fixed points. The values 0 and 1 will be used when processing the input/ output by merging operators to avoid the loss of the information.

By virtue of $\mathbf{G}$ and $\mathbf{H}$, the fuzziness of the primary terms and hedges are measured through (3.2), (3.3) and (3.4).

$$
\mathrm{fm}(\mathrm{S})=\theta=0,5 \cdot \mu(\mathrm{L})=\mu(\mathrm{V})=0,5 \rightarrow \alpha=\beta=0,5 \text { and } \mathrm{fm}(\mathrm{B})=1-\mathrm{fm}(\mathrm{S})=0,5 .
$$

In [6], the fuzzy terms are $\mathrm{AL}, \mathrm{AN}, \mathrm{Z}, \mathrm{DN}$ and $\mathrm{DL}$ of which $\mathrm{A}, \mathrm{D}, \mathrm{Z}$ stand for negative, positive and zero, respectively; whereas $\mathrm{N}$ and L stand for small and big, respectively. Now, the fuzzy terms are transformed to HA-terms:

$$
\mathrm{AL} \Rightarrow \mathrm{VS} \quad \mathrm{AN}=>\mathrm{LS} \quad \mathrm{Z} \Rightarrow \mathrm{W} \quad \mathrm{DN}=>\mathrm{LB} \quad \mathrm{DL} \Rightarrow \mathrm{VB}
$$

Step 3: The semantic representation of linguistic labels

The usual fuzzy rule base is converted into a linguistic rule base to convert the ordinary linguistic labels into HA-terms. By virtue of above parameters, (3.5) and (3.6), the FAM table is transfomed to SAM table. In fuzzy controller, the composition of fuzzy relations is presented via Table 3 [6], including linguistic values which describe inputs/ outputs $e_{i}, \dot{e}_{i}, u_{i}$ of controller. However, in hedge algebras, a set of rules is demonstrated by semantic Table 4 (m-SAM table), including the semantic values of the linguistic labels which describe $e_{i}, \dot{e}_{i}, u_{i}$. If inputs $e_{i}, \dot{e}_{i}$ 
belong to linguistic domains, with semantic value in $[0,1]$, then the controller will respond the output signal $\mathrm{u}_{\mathrm{i}}$ belong to the linguistic domain, with suitable semantic value to adjust $e_{i}, \dot{e}_{i}$ to 0 .

Table 3. FAM table (GTNN: linguistic values).

\begin{tabular}{|c|c|c|c|c|c|}
\hline GTNN & AL & AN & Z & DN & DL \\
\hline AL & DL & DL & DL & DN & Z \\
\hline AN & DL & DN & DN & Z & AN \\
\hline Z & DL & DN & Z & AN & AL \\
\hline DN & DN & Z & AN & AN & AL \\
\hline DL & Z & AN & AL & AL & AL \\
\hline
\end{tabular}

Table 4. m-SAM table (SV: semantic values).

\begin{tabular}{|c|c|c|c|c|c|}
\hline SV & $v(\mathrm{VS})=0,125$ & $v(\mathrm{LS})=0,375$ & $v(\mathrm{~W})=0,5$ & $v(\mathrm{LB})=0,625$ & $v(\mathrm{VB})=0,875$ \\
\hline$v(\mathrm{VS})=0,125$ & 0,875 & 0,875 & 0,875 & 0,625 & 0,5 \\
\hline$v(\mathrm{LS})=0,375$ & 0,875 & 0,625 & 0,625 & 0,5 & 0,375 \\
\hline$v(\mathrm{~W})=0,5$ & 0,875 & 0,625 & 0,5 & 0,375 & 0,125 \\
\hline$v(\mathrm{LB})=0,625$ & 0,625 & 0,5 & 0,375 & 0,375 & 0,125 \\
\hline$v(\mathrm{VB})=0,875$ & 0,5 & 0,375 & 0,125 & 0,125 & 0,125 \\
\hline
\end{tabular}

m-dimentional SAM table presents the semantic relationship between the input and the output; and, it represents a super surface in 3-D space. Merging operator will be used to exchange $\mathrm{m}$ dimensional SAM table to 2-dimensional SAM table which aims to simplify the interpolation. This paper use Product operator.

Table 5 represents 2-dimensional SAM table which is a curve in 2-D space $\mathrm{R}^{2}$. The horizontal axis is the integration of the semantic values of the linguistic labels which demonstrate $e_{i}$ and $\dot{e}_{l}$ and the vertical axis is the semantic value of the linguistic label which describes $u_{i}$. We can interpolate polynomial easily. In addition, a set of rules is chosen to be symmetry about semantics, so there is no point which has the similar horizontal degree with other ones.

No case in interpolation should be left out, if input's physical value is on the left of linguistic value AL's physical domain then its semantic value is $v(0)=0$. If input's physical value is on the right of linguistic value DL's physical domain then its semantic value is $v(1)=1$

Table 5. 2-SAM table.

\begin{tabular}{|c|c|}
\hline 0 & 1 \\
\hline 0.0156 & 0.875 \\
\hline 0.0469 & 0.875 \\
\hline 0.0625 & 0.875 \\
\hline 0.0781 & 0.625 \\
\hline 0.1094 & 0.5 \\
\hline 0.1406 & 0.625 \\
\hline 0.1875 & 0.625 \\
\hline 0.2344 & 0.5 \\
\hline 0.3281 & 0.375 \\
\hline 0.25 & 0.5 \\
\hline 0.3125 & 0.375 \\
\hline 0.4375 & 0.125 \\
\hline 0.3906 & 0.375 \\
\hline 0.5469 & 0.125 \\
\hline 0.7656 & 0.125 \\
\hline 1 & 0 \\
\hline
\end{tabular}


Step 4: Determining output's physical value

The controller's outputs are adjusted torques at actuated joints; however, the interpolation based on 2-dimensional SAM table which gives the semantic values of the linguistic variables. We need to determine real physical values of outputs via their semantics. To receive outputs' real physical values, we map their semantic values from $[0,1]$ to their individual physical domains.

The simulink model for HA-controller is presented in Figure 3.

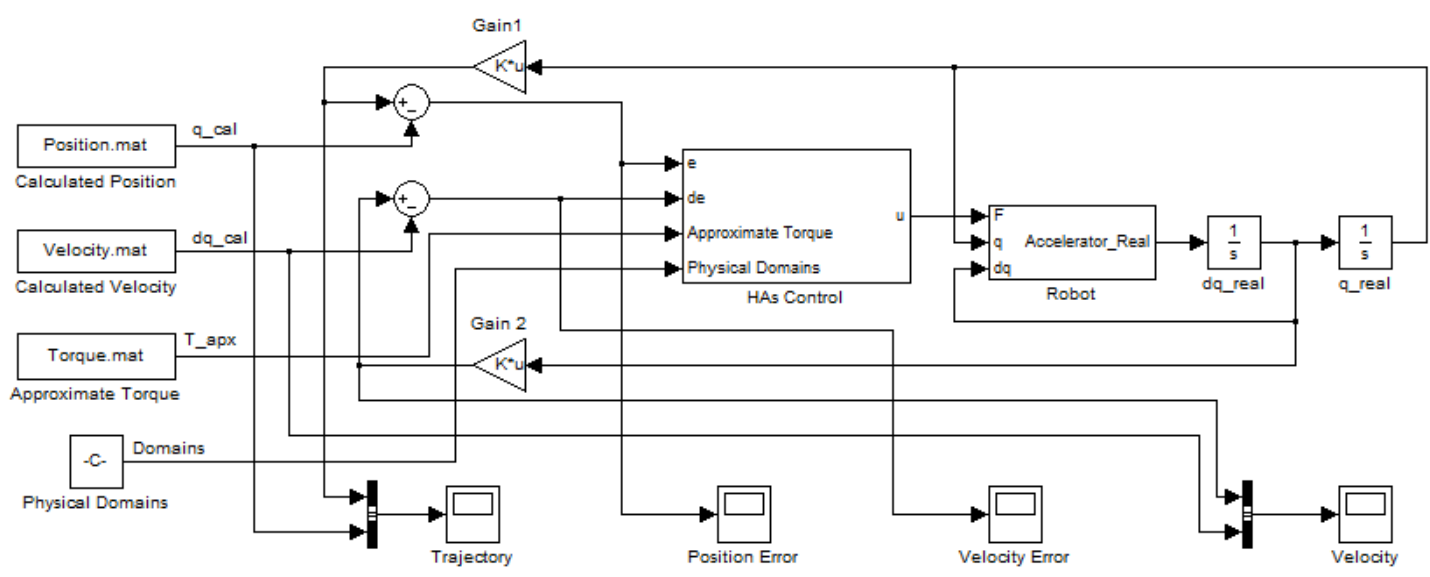

Figure 3. Simulink model for HA controller.

herein Position.mat and Velocity.mat are desired position and velocity which are obtained from kinematic problem and trajectory planning. Physical Domain is approximate domains of inputs and outputs, Torque.mat is approximate torque. HAs Control is controller via hedge algebras; Robot is the robot model, all are programmed in M-File which are inserted into Simulink by Matlab Function.

\section{SIMULATION RESULTS}

The proposed model of MRM robot is described in section (2); and, its kinematic and dynamical parameters are shown in Table 7. In this paper, the mass, the center of mass and the inertia tensor are received from design software. By doing this, the proposed model of MRM robot is more accurate and closer to real robot model (because robot's links are not homogeneous and its cross sections are not unchanged in reality). Based on the technical requirements in section (2.1) and robot's kinematics and dynamics, we obtain the results in Figure 4 - Figure 11 by applying above control methods.

Table 6. Physical domains of inputs/output of HA controller.

\begin{tabular}{|c|c|c|c|}
\hline Joint & $\mathrm{e}_{\mathrm{i}}$ & $\dot{e}_{\mathrm{i}}$ & $\mathrm{u}_{\mathrm{i}}$ \\
\hline 1 & {$[-0.002,0.002](\mathrm{rad})$} & {$[-0.06,0.06](\mathrm{rad} / \mathrm{s})$} & {$[-42,41.73](\mathrm{N} . \mathrm{m})$} \\
\hline 2 & {$[-0.002,0.002](\mathrm{rad})$} & {$[-0.09,0.09](\mathrm{rad} / \mathrm{s})$} & {$[-47,46.08](\mathrm{N} . \mathrm{m})$} \\
\hline 3 & {$[-0.0001,0.0001](\mathrm{m})$} & {$[-0.08,0.08](\mathrm{m} / \mathrm{s})$} & {$[-54.6,55](\mathrm{N})$} \\
\hline 4 & {$[-0.0001,0.0001](\mathrm{rad})$} & {$[-0.05,0.05](\mathrm{rad} / \mathrm{s})$} & {$[-68,68](\mathrm{N} . \mathrm{m})$} \\
\hline 5 & {$[-0.0001,0.0001](\mathrm{rad})$} & {$[-0.03,0.03](\mathrm{rad} / \mathrm{s})$} & {$[-8.0,8.1](\mathrm{N} . \mathrm{m})$} \\
\hline
\end{tabular}


Table 7. Kinematic and dynamical parameters of MRM robot.

\begin{tabular}{|c|c|c|c|c|c|c|}
\hline \multicolumn{2}{|c|}{ Link } & 11 & 12 & 13 & 21 & 22 \\
\hline \multicolumn{2}{|c|}{ Weight(kg) } & 8 & 8 & 3.5 & 50 & 20 \\
\hline \multirow{4}{*}{$\begin{array}{c}\text { Coordinates of links' } \\
\text { barycenter on local } \\
\begin{array}{c}\text { Cartesian coordinate } \\
\text { system }\end{array}\end{array}$} & $\mathrm{x}(\mathrm{m})$ & -0.165 & -0.165 & 0 & 0 & 0.068845 \\
\cline { 2 - 7 } & $\mathrm{y}(\mathrm{m})$ & 0 & 0 & 0 & 0 & 0.022064 \\
\cline { 2 - 7 } & $\mathrm{z}(\mathrm{m})$ & 0 & 0 & 0.2 & -0.077511 & 0.107575 \\
\cline { 2 - 7 } & $\mathrm{I}_{\mathrm{xx}}$ & 0.002818 & 0.002716 & 0.041672 & 1.36253 & 0.204889 \\
\cline { 2 - 7 } & $\mathrm{I}_{\mathrm{yy}}$ & 0.108298 & 0.115332 & 0.041672 & 1.146869 & 0.188627 \\
\cline { 2 - 7 } & $\mathrm{I}_{\mathrm{zz}}$ & 0.108825 & 0.115847 & 0.000166 & 1.861916 & 0.307898 \\
\cline { 2 - 7 } & $\mathrm{I}_{\mathrm{xy}}$ & 0 & 0 & 0 & 0 & 0.005476 \\
\cline { 2 - 7 } & $\mathrm{I}_{\mathrm{xz}}$ & 0.000381 & -0.00002 & 0 & 0 & 0.008992 \\
\cline { 2 - 7 } & $\mathrm{I}_{\mathrm{yz}}$ & 0 & 0 & 0 & 0 & -0.002919 \\
\hline
\end{tabular}

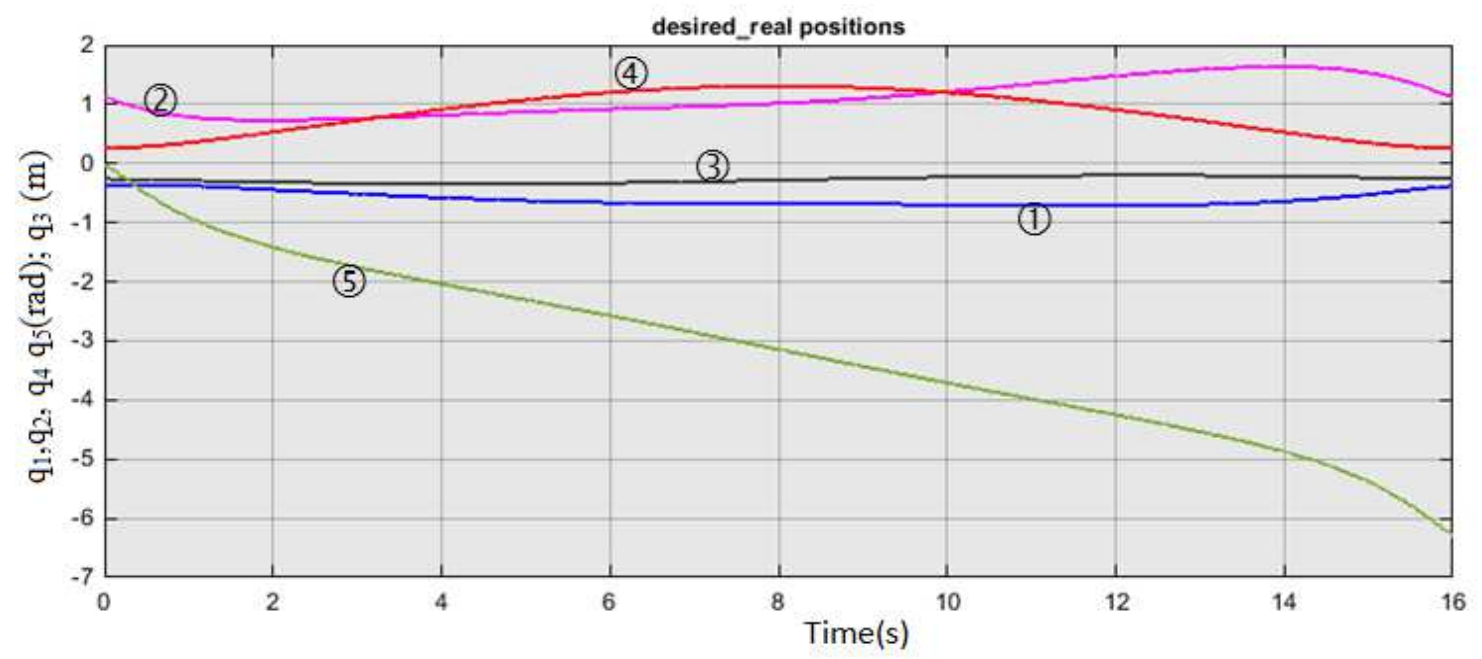

Figure 4. Graph of desired and real trajectory of MRM robot's 5 joints.

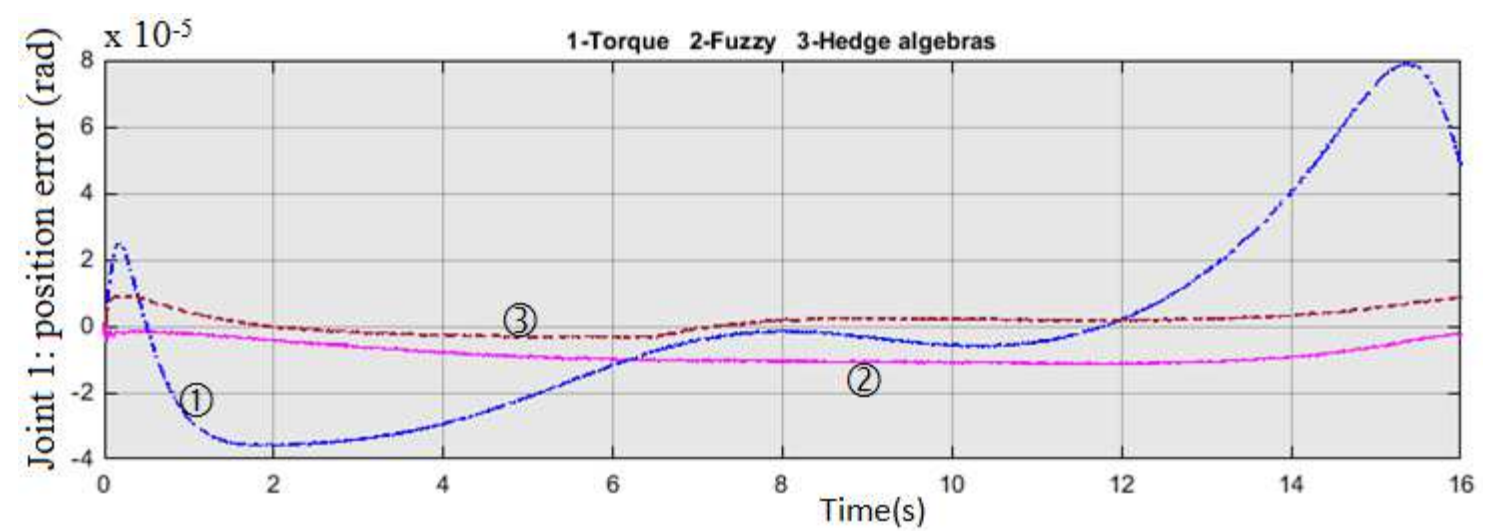

Figure 5. Graph of position error of the joint $11\left(\mathrm{e}_{1}\right)$. 


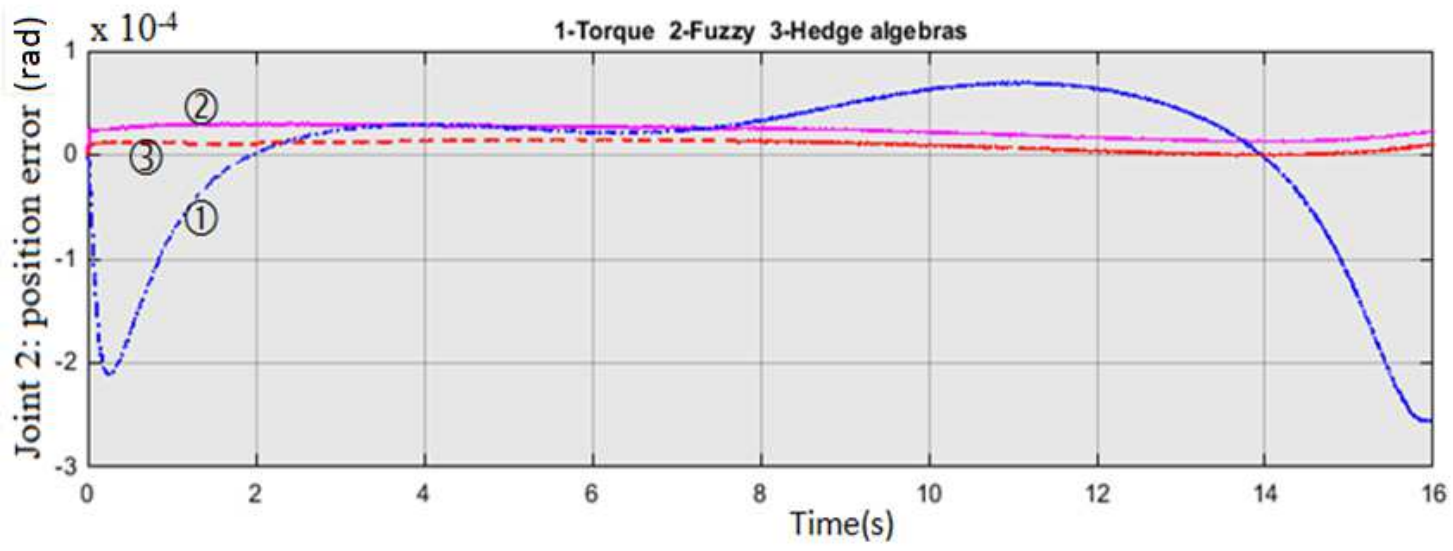

Figure 6. Graph of position error of the joint $12\left(\mathrm{e}_{2}\right)$.

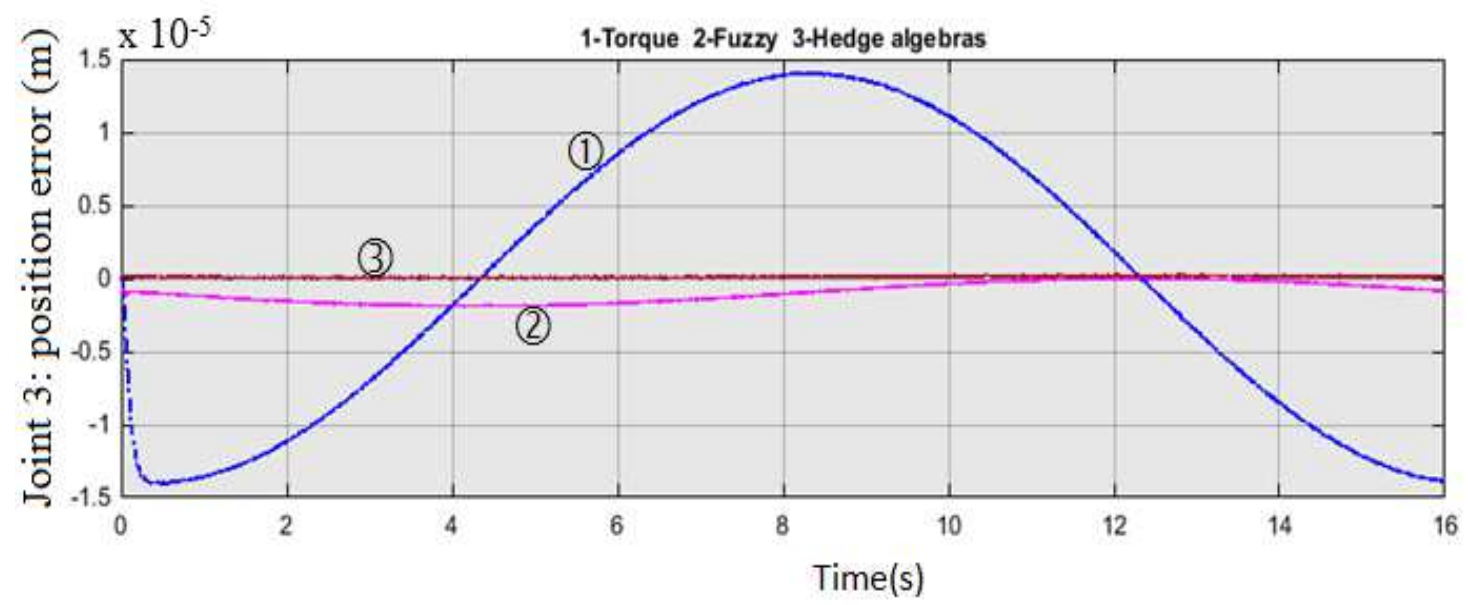

Figure 7. Graph of position error of the joint $13\left(\mathrm{e}_{3}\right)$.

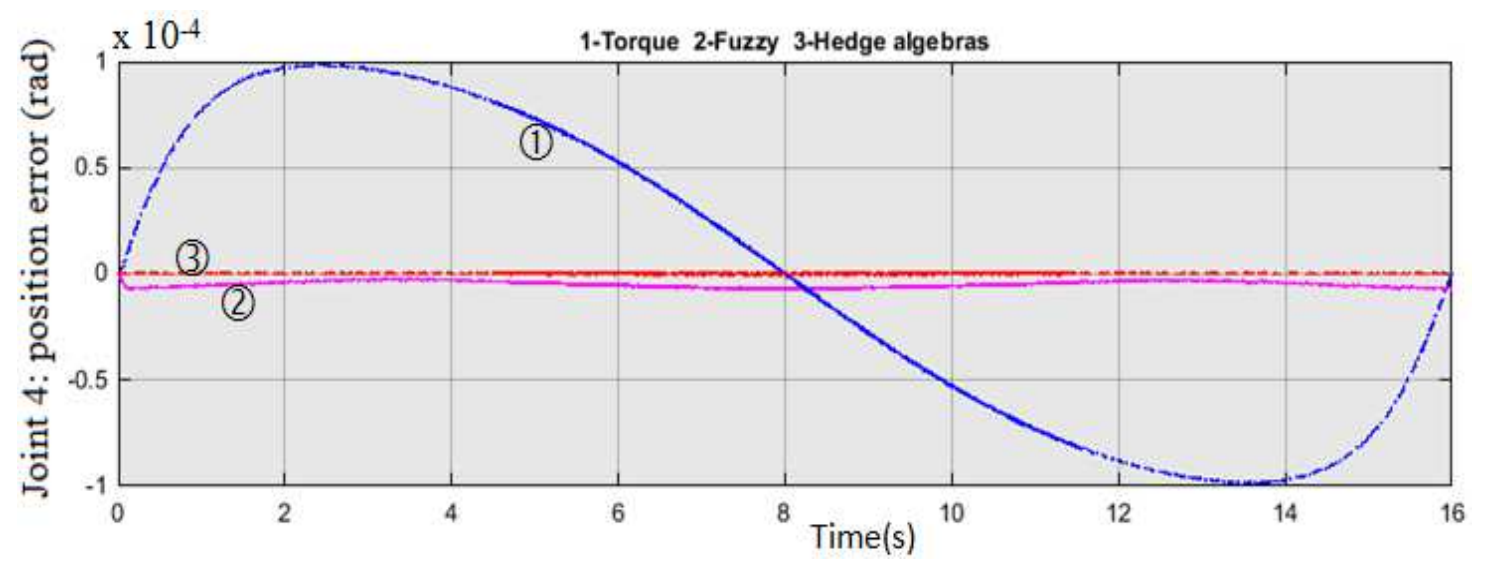

Figure 8. Graph of position error of the joint $21\left(\mathrm{e}_{4}\right)$. 


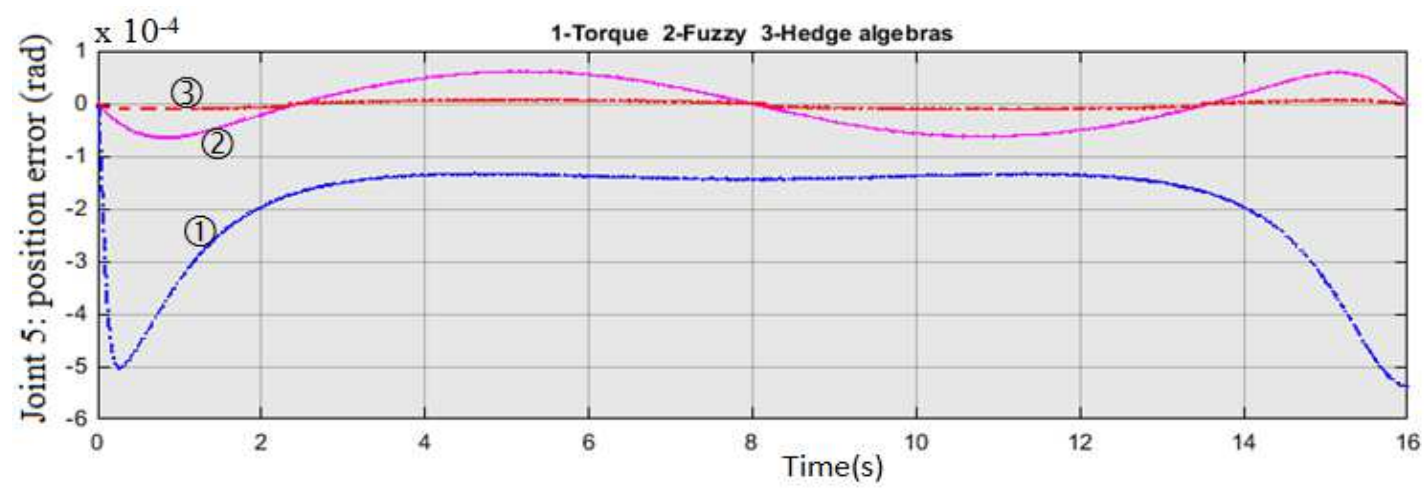

Figure 9. Graph of position error of the joint $22\left(\mathrm{e}_{5}\right)$.

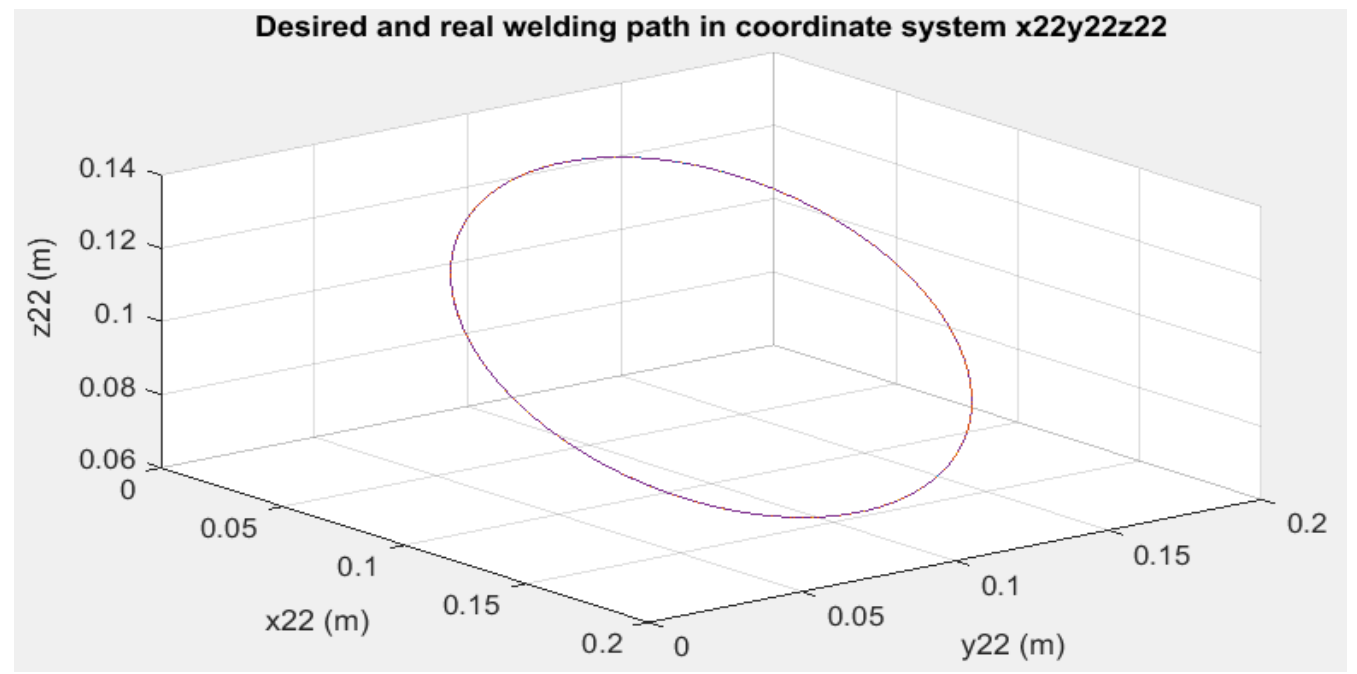

Figure 10. Desired and real welding path in Cartesian coordinate system $\mathrm{x}_{22} \mathrm{y}_{22} \mathrm{Z}_{22}$ of jig-robot's table (Fig. 1).

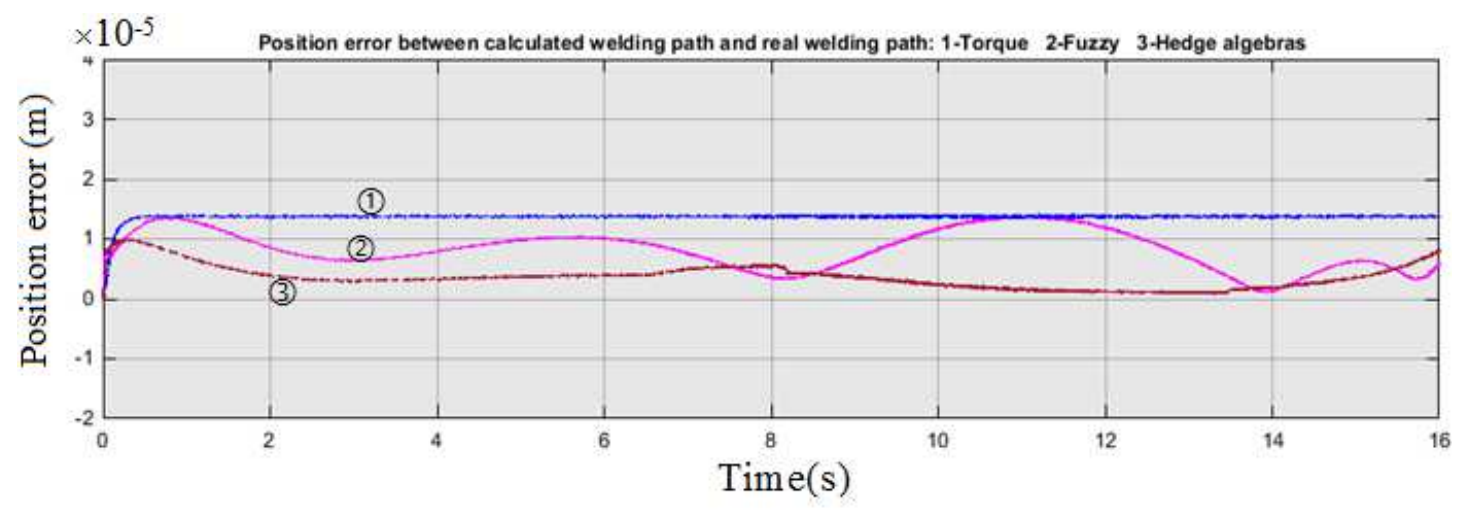

1-Torque, 2-Fuzzy, 3-Hedge algebras

Figure 11. Position error between calculated welding path and real welding path 
The desired and real welding path, received by torque, fuzzy and HAs controller overlap each other because the position error is very small (Fig. 10). Figure 11 shows the position error of each path.

\section{CONCLUSION}

The recevied results show that the proposed method is more accurate than the classical fuzzy method. Moreover, the application of HA in control will be easier to understand and simpler than the use of fuzzy logic. It brings several benefits for controlling engineering systems. This paper used 5 linguistic values for the fuzzification of inputs and outputs. To compare HA method with the fuzzy controller in the same condition, we just use the partition $\mathrm{k}=2$ for inputs/output. The analysis and results contribute a meaningful part in the expansion of research and application of modern theories for controlling robots which have complex structure, such as MRM robot. However, the use of Product operator to convert m-SAM table to 2-SAM table can lead to the loss of the control information, so this problem will be analyzed in the next studies.

\section{REFERENCES}

1. Phan Bui Khoi - Calculation and simulation of the program motion of mechanisms of relative manipulation (MRM), Journal of Science and Technology 3 (2009)19-28.

2. Phan Bui Khoi - Kinematic problems of programming control of mechanism of relative meanipulation, Proceedings of National Conference on Mechanics, Proceedings of National Conference on Mechanics 2 (2009) 317-323 (Vietnamese).

3. Afonin V. L., Phan Bui Khoi - Method for calculating action and constrained reactions forces in mechanisms of relative manipulation while executing programming motion, IMASH. RAS, Moscow, 1997 (Rusian).

4. Phan Bui Khoi- Applying Principle of Compatibility for analyzing forces of Mechanism of Relative Manipulation Robot. Thesis of PhD. IMASH. RAS, Moscow, 1997, in Rusian.

5. Phan Bui Khoi - Dynamical investigation of relation manipulation mechanisms in mechanical processing. Proceedings of National Conference on Mechanics 1 (2004) 181-190 (Vietnamese).

6. Phan Bui Khoi, Nguyen Van Toan - Application of fuzzy logic for controlling Mechanism of Relative Manipulation robot (MRM robot), Journal of Science and Technology 54 (3) (2016) 385-401.

7. Hai-Le Bui, Duc-Trung Tran, Nhu-Lan Vu (2011) - Optimal fuzzy control of an inverted pendulum. Journal of Vibration and Control 18 (14) 2097-2110.

8. Nguyen Dinh Duc, Nhu-Lan Vu, Duc-Trung Tran, Hai-Le Bui - A study on the application of hedge algebras to active fuzzy control of a seism-excited structure, Journal of Vibration and Control 18 (14) (2011) 2186-2200.

9. Anh N. D., Hai-Le Bui, Nhu-Lan Vu, Duc-Trung Tran - Application of hedge algebrabased fuzzy controller to active control of a structure against earthquake, Struct. Control Health Monit. 20 (2013) 483-495. 
10. Dinko Vukadinović, MateoBašić, CatHoNguyen, NhuLanVu, Tien DuyNguyen - Hedgealgebra-based voltage controller for a self-excited induction generator, Control Engineering Practice 30 (2014) 78-90.

11. Phan Anh Phong, Dinh Khac Dong, Tran Dinh Khang - Hedge Algebra based Type-2 Fuzzy Logic System and its Application to Predict Survival Time of Myeloma Patients, International Conference on Knowledge and Systems Engineering (2009) 13-18.

12. Nguyen Cat Ho, Huynh Van Nam - An algebraic approach to linguistic hedges in Zadeh's fuzzy logic, Fuzzy Sets and System 129 (2002) 229-254.

13. Nguyen Cat Ho, Wolfgang Wechler - Extended hedge algebras and their application to fuzzy logic, Fuzzy Sets and System 52 (1992) 259- 281.

14. Cat Ho Nguyen, Thai Son Tran, Dinh Phong Pham - Modeling of a sematics core of linguistic terams based on an extension of hedge algebra semantics and its application, Knowledge-Based Systems 67 (2014) 244-262.

15. Nguyen Cat Ho, Huynh Van Nam - A theory of refinement structure of hedge algebras and its application to fuzzy logic, Logic, algebra, and computer publications, Institute of mathematics, Polish Academy of Sciences Warszawa 46 (1999) 63-91.

16. Nguyen Cat Ho and W.Wechler - Hedge algebras: An algebraic approach to structure of sets of linguistic truth values, Fuzzy Sets and Systems 35 (1990) 281-293.

17. Do Sanh - On the principle of compatibility and the equation of a constrained mechanical system, ZAMM (1980) 210-212.

18. Do Sanh - On the motion of controlled mechanical system. Advances in Mechanics 7(2) (1984) 3-24. 